\title{
Role of NF-k $\beta$ factor Rel2 during Plasmodium falciparum and bacterial infection in Anopheles dirus
}

\author{
Mohammad Behram Khan, Jonathan Wee Kent Liew, Cherng Shii Leong and Yee-Ling Lau*
}

\begin{abstract}
Background: Anopheles mosquitoes transmit malaria which is one of the world's most threatening diseases. Anopheles dirus (sensu stricto) is among the main vectors of malaria in South East Asia. The mosquito innate immune response is the first line of defence against malaria parasites during its development. The immune deficiency (IMD) pathway, a conserved immune signaling pathway, influences anti-Plasmodium falciparum activity in Anopheles gambiae, An. stephensi and An. albimanus. The aim of the study was to determine the role of Rel2, an IMD pathway-controlled NF-kappaß transcription factor, in An. dirus.

Methods: RACE (Rapid amplification of cDNA ends) was performed on the Rel2 gene. Double-stranded Rel2 was constructed and injected into the thorax of female mosquitoes. The injected mosquitoes were fed on a $P$. falciparum gametocyte culture and dissected on day 7-9 post-feeding in order to count the oocysts. A survival analysis was conducted by exposing the dsRNA injected mosquitoes to Gram-positive and Gram-negative bacteria.

Results: This study demonstrated that the Rel2 gene in An. dirus has two isoforms, short length and full length. RNA interference-mediated gene silencing of Rel 2 showed that the latter is involved in protection against $P$. falciparum, Gram-positive bacteria (Micrococcus luteus) with Lys-type peptidoglycan and Gram-negative bacteria (Escherichia coli) with DAP-type peptidoglycan.

Conclusion: This study suggested that there are similarities in the splicing events and functionality of the Rel2 gene, between the Anopheles species. Among all the important anophelines, the immunity of only a few has been thoroughly investigated. In order to develop novel vector-based control strategies and restrict malaria transmission, the immune pathways of these important vectors should be thoroughly investigated.
\end{abstract}

Keywords: Anopheles dirus, IMD pathway, Rel2, Plasmodium falciparum, Bacterial infection

\section{Background}

Malaria, caused by the protozoan parasites Plasmodium spp., is among the world's most life threatening infectious diseases. About 70 species of Anopheles are capable of transmitting malaria [1]. Nearly 2 billion humans are at risk of contracting malaria in the South East Asia Region (SEAR) [2]. Anopheles dirus (sensu stricto) (s.s) (species A) is an important vector and can be found in Thailand, Myanmar, Cambodia, Vietnam and Laos [3]. The highly anthropophilic nature of $A n$. dirus $A$ is also one of the reasons for this species being the main malaria vector in

\footnotetext{
* Correspondence: lauyeeling@um.edu.my

* Correspondence: lauyeeling@um.edu.my Lumpur, Malaysia
}

(c) 2016 The Author(s). Open Access This article is distributed under the terms of the Creative Commons Attribution 4.0 International License (http://creativecommons.org/licenses/by/4.0/), which permits unrestricted use, distribution, and reproduction in any medium, provided you give appropriate credit to the original author(s) and the source, provide a link to the Creative Commons license, and indicate if changes were made. The Creative Commons Public Domain Dedication waiver (http://creativecommons.org/publicdomain/zero/1.0/) applies to the data made available in this article, unless otherwise stated. quired to curb the disease, especially in light of rece problems that include drug-resistant parasites and insecticide-resistant mosquitoes [5].

Insect immunity exclusively relies on the innate immune response and is devoid of the adaptive immune response. The model organism for the insect immunity, Drosophila melanogaster, has provided insights on the two main regulatory pathways, Toll and IMD. In Drosophila, the NF-k $\beta$ factors associated with the Toll pathway are Dif and Dorsal, whereas Relish ( $R e l)$ is involved in the IMD pathway [6, 7]. In An. gambiae, Rel1 is an analogue of Dif, and Rel2 is the orthologue of the Relish gene [8,9]. The most effective anti-Plasmodium immune factors, TEP1, APL1, LRRD7 and 
FBN9, are all regulated by the NF-k $\beta$ Rel2 transcription factor [10-13]. In Anopheles, the Rel2 gene undergoes alternative splicing to produce a full-length form (Rel2-F) and a shorter form (Rel2-S). The full-length form consists of the Rel-homology Domain (RHD), carboxyl-terminal ankyrin (ANK) and death domains, whereas the shorter form lacks these inhibitory domains.

The Drosophila IMD pathway is activated against Gramnegative bacteria and some Gram-positive bacilli, which contain diaminopimelic acid (DAP-type) in their peptidoglycan (PGN). On the other hand, fungi and Gram-positive bacteria with lysine (Lys-type) in the PGN switch on the Toll pathway $[6,14]$. The PGN-recognition proteins (PGRPs) in An. gambiae and Ae. aegypti activate the IMD pathway, irrespective of the type of PGN present [15] [16].

In this study, we elucidated the role of Rel2 in $A n$. dirus (s.s) by using RNA interference (RNAi) during Plasmodium falciparum and bacterial infections.

\section{Methods}

\section{Mosquito rearing}

Anopheles dirus A (WRAIR2) strain from Malaria Research and Reference Reagent Resource Center (USA) was colonized in the lab using the forced copulation technique. The mosquitoes were maintained at $25{ }^{\circ} \mathrm{C}$, relative humidity of $85 \%$, with a 12 -h light/dark photocycle and fed on a $10 \%$ sucrose solution.

\section{Rel2 primer design}

A short conserved region of $\operatorname{Rel} 2$ (241 bp) was amplified by designing primers based on the GenBank sequence for An. quadriannulatus (Accession No. EU304622.1), An. coluzzi (Accession No. KP274426.1), An. gambiae M isolate (Accession No. GU990219.1) and An. merus (Accession No. KP274430.1). Rel2 forward and reverse primer sequences: 5'-CAA GTT TCG CTT CCG CTA CCA G-3' and 5' -ACC CAC ATC CAG ATC GTG-3'.

\section{Rapid amplification of CDNA ends (RACE)}

Total RNA from adult female mosquitoes was extracted using ReliaPrep ${ }^{\text {Tw }}$ RNA Tissue Miniprep System (Promega, Fitchburg, USA) according to the manufacturer's instructions. The extracted RNA was used as the template in SMARTer $^{\mathrm{mm}}$ RACE cDNA amplification kit (Clonetech, Mountain View, USA) to obtain the full cDNA sequence. The sequence obtained from Rel2 PCR was utilized in designing primers for RACE. The gene specific primers (GSP) and nested gene specific primers (NGSP) used to amplify the $5^{\prime}$ and $3^{\prime}$ RACE products are shown in Table 1.

\section{RNAi gene silencing}

The sequence obtained from RACE was used to construct double-stranded RNA using the T7 Megascript Kit (Ambion, Foster City, USA). A 445 bp Rel2 region was amplified and the amplicon was ligated downstream of the T7 promoter in the pGEM ${ }^{\circ}-\mathrm{T}$ Vector (Promega) to produce sense and antisense RNA strands. Approximately $50-200 \mathrm{nl}$ of dsRNA $(2 \mu \mathrm{g} / \mu \mathrm{l})$ was injected into the thorax of anesthetized 2-4 days old female mosquitoes by using InjectMan ${ }^{\bullet}$ NI 2 (Eppendorf, Hamburg, Germany) and FemtoJet ${ }^{\circ}$ express (Eppendorf) with Femtotips $^{\circ}$ I (Eppendorf) microcapillaries. The dsLacZ (441 bp) was used in the control injection. The primers used for the construction of dsRel 2 and dsLacZ are shown in Table 1.

\section{Reverse transcription-qPCR}

The cDNA was synthesized using the QuantiTect ${ }^{\circ}$ Reverse Transcription (Qiagen, Hilden, Germany) according to the manufacturer's instructions. A reverse transcription quantitative PCR was carried out using the $\mathrm{iQ}^{\mathrm{Tm}} \mathrm{SYBR}^{\circ}$ Green Supermix (Bio-Rad, Hercules, USA) on CFX96 ${ }^{\text {th }}$ RealTime System, $\mathrm{C} 1000^{\mathrm{im}}$ Thermal cycler. The primers are shown in Table 1. All qPCR reactions were performed in triplicates. The melting curves were obtained for each data point to validate the specificity of the PCR reactions. The expression level of Rel2 in dsRel2-treated samples was compared to that of $d s L a c Z$-treated samples by normalizing the cDNA levels using the ribosomal protein $S 7$ gene.

\section{Gametocyte culture of $P$. falciparum and mosquito infection}

Gametocytogenesis in the NF54 strain of P. falciparum was induced using an established protocol [17]. On day 16 , the culture was pelleted and resuspended in an equal volume of heat-inactivated human serum before transferring to a membrane feeder set at $37{ }^{\circ} \mathrm{C}$, and fed to mosquitoes (injected with dsRNA on day 12 of the $P$. falciparum culture). The fully engorged female mosquitoes were maintained at $25{ }^{\circ} \mathrm{C}$ for $7-9$ days. Mosquitoes

Table 1 Primers used for rapid amplification of cDNA ends, RTqPCR and dsRNA

\begin{tabular}{ll}
\hline Primer & Sequence 5'-3' \\
\hline RACE 5' Rel2 GSP & CGCTTCTGCGGGTCCACCTGGTACAGGGG \\
RACE 5' Rel2 NGSP & CAGCGAGCCGTGCGTACCGTGCATCTCC \\
RACE 3' Rel2 GSP & GCACGGTACGCACGGCTCGCTGATGGGC \\
RACE 3' Rel2 NGSP & CGCCGGTGAGGCTAAGGTGCGCTGCTCC \\
RT-qPCR S7-F & AGAAGAAGTTCTCCGGTAAG \\
RT-qPCR S7-R & CGGTCTCTTCTGCTTGT \\
RT-qPCR Rel2-F & GGCAGGACAATCTACAAAC \\
RT-qPCR Rel2-R & CTCCAGGATCACGAGATAG \\
dsRel2-F & CCCTACTGAATTTGGACAGC \\
dsRel2-R & ATTTGCTGCTGTTGGGACTG \\
dsLaCZ-F & CATTACCAGGCCGAAGCAG \\
dsLaCZ-R & GCGGCTGATGTTGACTGGAAG \\
\hline
\end{tabular}


that survived were dissected and the midguts were stained with mercurochrome to check for oocysts. Analysis of the infection prevalence was performed using the Chi-square test whereas for oocyst infection intensity, it was determined by the Mann-Whitney $U$ test. The experiment was performed with at least 3 biological replicates and the data were pooled if there was no significant difference between the replicates within the dsRNA treatments.

\section{Bacterial challenge}

Escherichia coli and $M$. luteus were cultured to $\mathrm{OD}_{600}$ 0.7 , pelleted, washed and resuspended in PBS. The final $\mathrm{OD}_{600}$ for infection was 0.4 for $E$. coli and 0.1 for $M$. luteus. Four days after the dsRNA injection, mosquitoes were infected with the bacteria by inserting Femtotips ${ }^{\circledR} \mathrm{I}$ (Eppendorf) microcapillary in the bacteria culture and poking it in the mosquito's thorax. The adult mosquitoes that did not recover after $3 \mathrm{~h}$ of being exposed to the bacteria were not considered for the analysis. Dead mosquitoes were counted daily and recorded for 10 days. At least three independent experiments were performed, each with at least 25 female mosquitoes per group. The survival analysis was performed using Kaplan-Meier logrank test.

\section{Results}

\section{Rel2 knockdown efficiency}

Four days after the dsRNA injection, RNA was extracted and converted to cDNA to be used in reverse transcription quantitative PCR. The primers shown in Table 1 were used to determine the Rel2 silencing efficiency. The $2_{\mathrm{T}}^{-\Delta \Delta \mathrm{C}}$ method was used to determine the relative gene expression of Rel2 with $S 7$ as an endogenous reference gene. At least triplicates were performed in the RTqPCR reaction. The knockdown efficiency calculated was $64.6 \%$ (Fig. 1).

\section{Knockdown of Rel2 increases susceptibility to $P$. falciparum infection in An. dirus}

In order to investigate the function of Rel2, an RNAi mediated gene silencing approach was used. RACE results showed that Rel2 has two transcripts: a short (Rel2S) and a full-length form (Rel2-F). The length of the short form (Rel2-S) was around $1.7 \mathrm{~kb}$ whereas the full length (Rel2-F) covered approximately $3.2 \mathrm{~kb}$. Since dsRNAs act only against mRNAs that contain the corresponding sequence [18], the $d s R e l 2$ construct was designed to silence both forms of the gene by targeting the $5^{\prime}$ region of the short and full length transcripts.

The strain of $P$. falciparum used for infection in this study did not have an unnaturally high infection level in the mosquito [19]. In order to obtain a substantial infection, the $P$. falciparum culture at $0.3 \%$ gametocytemia was fed to the mosquitoes [20]. A higher gametocytemia $(\geq 2 \%)$ resulted in infection rates that were undetectable in both groups of dsRNA-treated mosquitoes.

Time point gene expression experiments conducted at 2, 12, 24 and $48 \mathrm{~h}$ post $P$. falciparum blood-feeding showed that Rel2 is upregulated the most (1.94-fold) at the $2 \mathrm{~h}$ interval (Additional file 1: Figure S1). Silencing the IMD pathway factor, Rel2, produced a phenotype that led to a significant increase in infection prevalence as compared to the $d s L a c Z$-treated controls $\left(\chi^{2}=5.39, d f=1, P=\right.$ 0.020; Fig. 2). The $d s L a c Z$-treated controls had a median oocyst number of 1 , with infection prevalence of $21.4 \%$, while the $d s R e l 2$-treated mosquitoes had a 2-fold greater median oocyst number of 2 and an infection prevalence of $37.2 \%$. Furthermore, the knockdown of Rel2 also resulted in significantly higher oocyst infection intensity (MannWhitney $U=144.0, P=0.0013$; Fig. 3).

\section{Rel2 is required against bacterial infection in An. dirus}

The potential role of Rel2 in the defence against bacterial infection in adult mosquitoes was investigated through RNAi analysis. Two to four day-old female mosquitoes were injected with dsRNA and exposed to $E$. coli (Gram-negative) and M. luteus (Gram-positive) after 4 days. RT-qPCR was used to confirm the knockdown of the Rel2 transcript.

The preliminary infection experiments suggested the use of O.D $D_{600}=0.4$ for E. coli $[21]$ and O.D D $_{600}=0.1$ for $M$. luteus. The mosquito survival study was conducted for 10 days after the bacterial infection. Rel2 knockdown severely compromised the survival of the adult mosquitoes when exposed to $E$. coli $\left(\chi^{2}=8.19, d f=1, P=0.004\right.$; Fig. 4a). A similar trend was observed during the $M$. luteus infection in the Rel2-silenced mosquitoes when compared to the $d s L a c Z$-treated controls $\left(\chi^{2}=5.05, d f=\right.$ $1, P=0.025$; Fig. 4b).

\section{Discussion}

It is relatively much more convenient to conduct experiments on naturally mating strains compared to colonies maintained through forced copulation. The degree of penetration of the microcapillary in the thorax of the mosquito had an immense effect on the mortality rate, post-injection. Adept and experience in the use of the micromanipulator improves mosquito survival rate to more than approximately $80 \%$. The limitation during the injection process was the varying volume of dsRNA injected; the volume was estimated by dividing the volume of dsRNA loaded in the microcapillary with the total number of injections possible, at the set parameters.

In the Drosophila model system, Dredd cleaves the ankyrin repeat region of the Relish gene, resulting in the translocation of the active form of Relish into the 


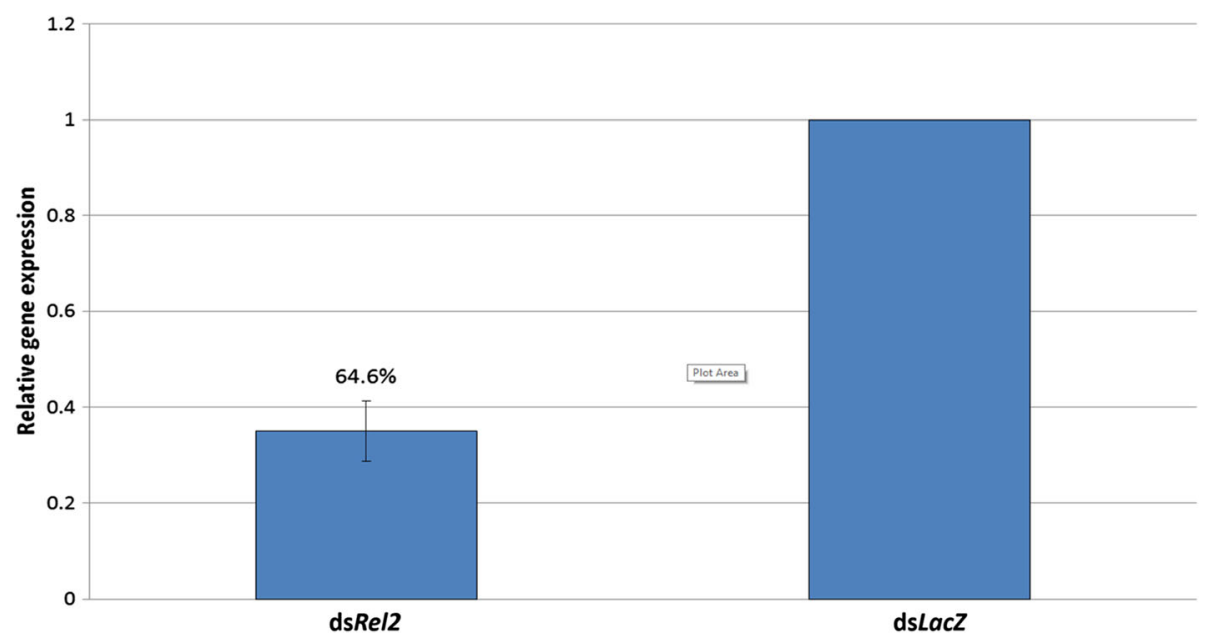

Fig 1 dsRNA mediated silencing efficiency of Rel2. The $2_{T}^{-\Delta \Delta C}$ method was utilized to determine the relative gene expression from qPCR data with S7 as an endogenous reference gene. The silencing efficiency of Rel2 knockdown was $64.6 \pm 6.3 \%$

nucleus to activate the IMD pathway [22]. In Aedes, the orthologue of Relish, Rel2, has one additional alternatively spliced transcript than that of Anopheles, encoding only the ANK domain [23]. Since Rel2-S cannot be targeted specifically without affecting Rel2-F, the distinct roles of each are still not evident. However, the Rel2-F can be independently silenced to elucidate the function by targeting the region encoding the ankyrin repeats. One study reported Rel2-F as having no effect on the prevalence of $P$. falciparum infection [10]. Hemozoin treatment was shown to induce the full length form of Rel2 and reduce P. berghei infection in An. gambiae [24]. Garver and colleagues showed that Rel2-F had a mild effect on P. falciparum oocyst intensity in An. gambiae, which was not as potent as the Rel2-S and Rel2-F combination [25]. Even though the Toll and Jak-Stat pathways are involved in providing protection against $P$. berghei, P. falciparum and P. vivax [26-29], the IMD pathway has come out as the most effective against human malaria [25]. Our results also demonstrated that dsRel2-treated An. dirus mosquitoes have a significantly higher $P$. falciparum prevalence and oocyst infection intensity. Anopheles gambiae was shown to have an average of 2.2 oocysts per midgut after being infected with P. falciparum [30]. The knockdown of Rel2 in the Ngousso mosquito strain showed an increased susceptibility to $P$. falciparum in terms of infection prevalence [10]. Silencing the negative regulator (Cactus) of Rel2 or overexpressing the Rel2 transcription factor provides nearly complete refractoriness in laboratory reared $A n$.

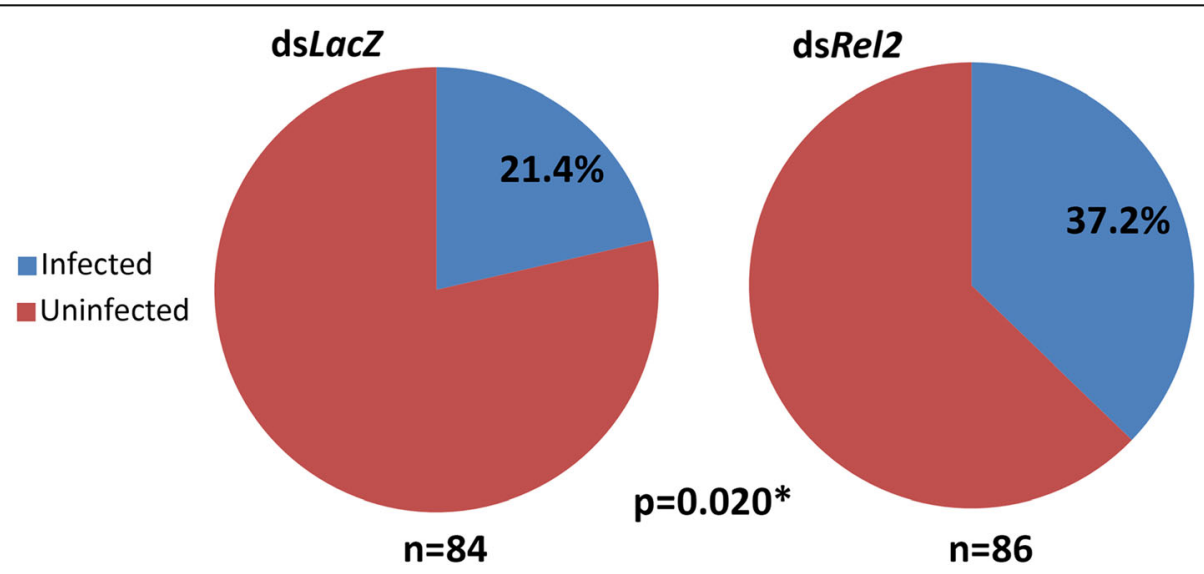

Fig 2 Anopheles dirus utilizes Rel2 against cultured Plasmodium falciparum. Silencing of the Rel2 gene showed significantly higher infection prevalence in An. dirus fed on cultured P. falciparum gametocytes. The dsLacZ-treatedted control had an infection prevalence of $21.4 \%$ whereas the $d s R e l 2$ injection in the mosquitoes resulted in a significantly higher prevalence of $37.2 \%\left(X^{2}=5.39, d f=1, P=0.020\right)$ 


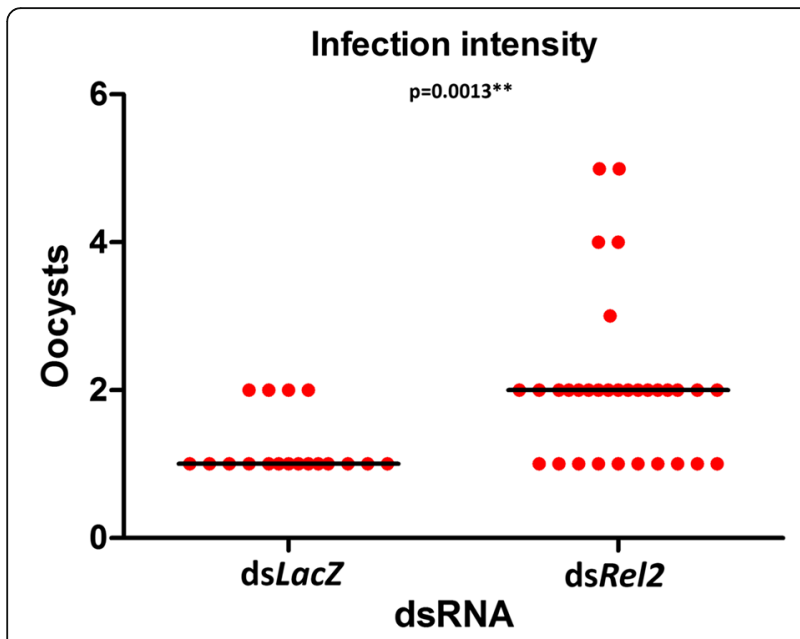

Fig 3 Rel2 restricts development of Plasmodium falciparum in Anopheles dirus. Oocyst infection intensity was significantly higher in dsRel2-treated mosquitoes compared to those with dsLacZ injected. The dots represent the individual oocyst and the horizontal bars represent the median number of oocysts detected. The P-value was calculated using the Mann-Whitney statistical test. (Mann-Whitney $U=144.0, P=0.0013$ )

gambiae, An. stephensi and An. albimanus against cultured P. falciparum [27]. The overexpression of Rel2 in the midgut tissue of An. stephensi following a blood meal also resulted in inefficient invasion of the ookinetes [20]. Silencing Imd, Fadd, Dredd and Rel2 had a significant effect on the infection intensity, but only the silencing of Rel2 had a significantly higher infection prevalence [25].

An upper limit for gametocyte density exists, beyond which the infection rates plummet [31, 32]. It seems that the low gametocyte density is an evolutionary mechanism to guarantee fertilization. The high gametocyte densities are extremely rare and probably of little significance epidemiologically. This explains the absence of oocysts when the mosquitoes were fed at a higher gametocytemia.

The position of the primer plays a pivotal role in detecting the accuracy of silencing in a given sample [33] because some primer sets may be able to bind to the cleaved products and amplify them, resulting in an underestimation of the silencing efficiency [34]. The primers targeting the 3 ' end of the mRNA might give false negative results, probably because of the presence of secondary structures or RNA binding proteins [35]. Therefore in this study, the primers were designed away from the 3' end of the Rel2 mRNA and downstream of the region targeted by the dsRel2 (Fig. 5). However, it is not always possible to cover a specific region of the mRNA because of the thermodynamic limitations posed in primer design for $\mathrm{RT}-\mathrm{qPCR}$.

Our results help to explore whether the role of Rel2 is conserved among different malaria vectors. There may well be differences among the anopheline species in some elements of these downstream mechanisms, but the collective effect results in an anti-Plasmodium falciparum immune response; the presence of multiple factors working in tandem would make it highly unlikely for P. falciparum to acquire resistance against this pathway [27].

In Drosophila, activation of the Toll or the IMD pathway depends on the detection of the PGN on the bacterial surface by the PGRPs. The Toll pathway is responsible when the Lys-type PGN is recognised by the PGRP-SA and PGRP-SD, whereas the IMD pathway is activated upon recognition of the DAP-type PGN by the PGRP-LC. However, the PGRP-LC in An. gambiae acts as the main receptor for Lys-type and DAP-type PGN and activates the IMD pathway via translocation of Rel2-F and Rel2-S into the nucleus, respectively [16]. RNAi-mediated knockdown of Rel2 in Ae. aegypti demonstrated a greater mortality to Gram-negative bacteria Enterobacter cloacae and

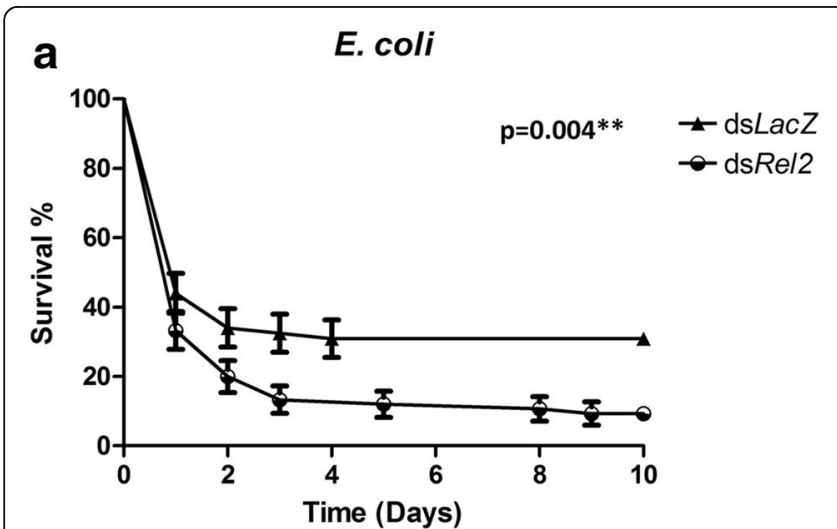

b

M. Iuteus

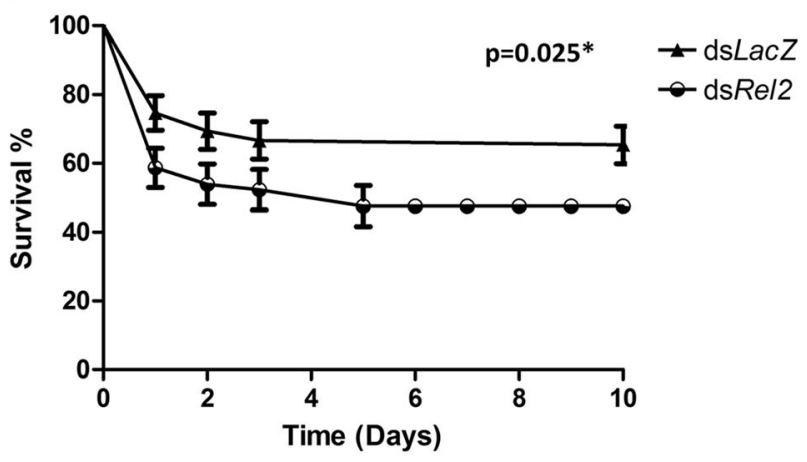

Fig 4 Rel2 provides protection against Gram-negative and Gram-positive bacteria. Kaplan-Meier survival analysis of dsRNA-treated mosquitoes after being infected with $\mathbf{a}$ Escherichia coli and $\mathbf{b}$ Micrococcus luteus. Each experiment was performed in triplicate with at least 25 mosquitoes per group. The vertical represent standard errors 


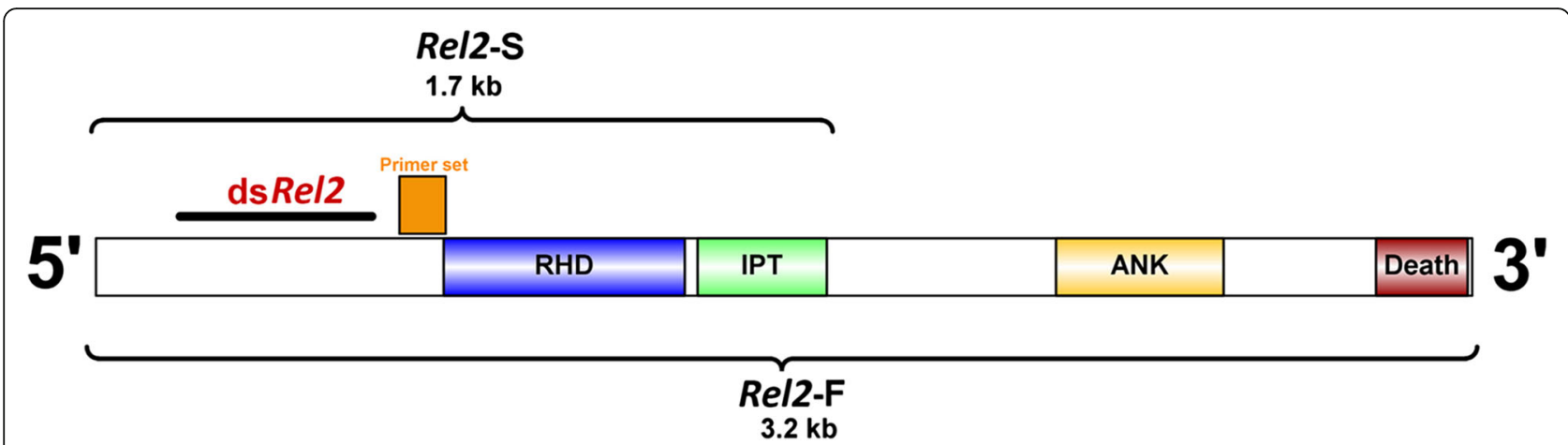

Fig 5 Schematic diagram of Rel 2 and the positions of dsRel 2 and RT-Rel 2 primers. The dsRel 2 primers targeted a 445 bp region near the $5^{\prime}$ end of the Rel2-S and Rel2-F isoforms. The RT-Rel2 primer set amplified a region of $107 \mathrm{bp}$, downstream of the dsRel2 target region and away from the 3' end of the gene

the Gram-positive Enterococcus faecalis [15]. Our results showed that Rel2 provides protection against DAP-type Gram-negative and Lys-type Gram-positive bacteria. Since the AMP genes such as Defensin, Cecropin, and Gambicin can be induced and regulated by both, Rel1 and Rel2 [36], it is possible that Rel1 can contribute to providing protection against certain PGNs, albeit at a lesser extent than Rel2. Since the dsRel2 in this study targeted the common domains of the Rel2-S (short form) and Rel2-F (full length), there is a possibility that the Rel2-F also has the potential to act against the pathogen/parasite. Therefore, in order to investigate the function of Rel2-F, it has to be independently targeted.

\section{Conclusions}

Although all human malaria species of Plasmodium are found in SEAR, P. falciparum and P. vivax are the most prevalent, with the latter species contributing more to the clinical cases currently [37]. However, the biggest threat in this region remains the emergence of $P$. falciparum resistance to artesunate [38]. In SEAR, the number of anopheline species poses a serious problem for malaria control programmes. The results presented in this study explore the role of Rel2 in an important vector in SEAR and adds to the growing information about mosquito immunity. Evidence from previous studies and this one portrays the significance of Rel2 against P. falciparum and bacterial infection. Nonetheless, Rel2 has the tendency to affect non-immune genes and the upregulation of the transcription factor may inadvertently have an impact on mosquito fitness and fecundity [39]. Further research has to be conducted to fully comprehend the ramifications of altering the natural gene expression of the mosquito. A thorough investigation of the JakSTAT, Toll, and IMD pathway in the anopheline species would serve as stepping stone towards generating parasite resistant mosquitoes.

\section{Additional file}

Additional file 1: Figure S1. Rel2 gene expression analysis. The mRNA expression level of Rel2 at different time points after being exposed to $P$. falciparum mature gametocytes. The $2 \mathrm{~h}$ interval depicted the highest fold change (1.94-fold) compared to the other time points. The vertical lines represent the standard deviation. (JPG $199 \mathrm{~kb}$ )

\section{Abbreviations \\ ANK: Ankyrin; ATCC: American type culture collection; dsRNA: Double- stranded RNA; GSP: Gene specific primers; IMD: Immune deficiency; IPT: Immunoglobulin-like, plexins, transcription factors; NGSP: Nested gene specific primers; PGN: Peptidoglycan; PGRPs: PGN-recognition proteins; Rel: Relish; RHD: Rel-homology domain; RNAi: RNA interference; s.s: sensu stricto}

\section{Acknowledgement}

We would like to thank ATCC for providing An. dirus WRAIR2 live eggs and Plasmodium falciparum NF54 strain.

\section{Funding}

This study was funded by University of Malaya High Impact Research Fund (UM.C/HIR/MOHE/MED/16) from the Ministry of Higher Education, Malaysia. The funders had no role in study design, data collection and analysis, decision to publish, or preparation of the manuscript.

\section{Availability of data and material}

The datasets supporting the results are included within the article and its Additional file 1. The partial Rel2 gene sequence data has been deposited in the GenBank database under accession number KU601345.

\section{Authors' contributions}

MKB and YLL conceived and designed the study. MBK, JKL and CSL performed the experiments. MBK and YLL analyzed the data, drafted the manuscript and helped in manuscript revision. All authors read and approved the final manuscript.

\section{Competing interests}

The authors declare that they have no competing interests.

\section{Consent for publication}

Not applicable.

\section{Ethics approval and consent to participate}

The study was carried out in strict accordance with the recommendations in the Guide for the Care and Use of Laboratory Animals of the National Institutes of Health. The protocol was approved by Institutional Animal Care and Use Committee, Faculty of Medicine, University of Malaya with the ethics number: 20150407/PARA/R/MBK. 
Received: 17 April 2016 Accepted: 21 September 2016 Published online: 29 September 2016

\section{References}

1. Hanafi-Bojd AA, Azari-Hamidian S, Vatandoost H, Charrahy Z. Spatiotemporal distribution of malaria vectors (Diptera: Culicidae) across different climatic zones of Iran. Asian Pac J Trop Med. 2011;4(6):498-504

2. Bharati K, Ganguly NK. Tackling the malaria problem in the South-East Asia Region: need for a change in policy? Indian J Med Res. 2013;137(1):36-47.

3. Obsomer V, Defourny P, Coosemans M. The Anopheles dirus complex: spatial distribution and environmental drivers. Malar J. 2007:6:26.

4. Trung HD, Bortel W, Sochantha T, Keokenchanh K, Briet OJ, Coosemans M. Behavioural heterogeneity of Anopheles species in ecologically different localities in Southeast Asia: a challenge for vector control. Trop Med Int Health. 2005;10(3):251-62.

5. Cirimotich CM, Clayton AM, Dimopoulos G. Low- and high-tech approaches to control Plasmodium parasite transmission by Anopheles mosquitoes. J Trop Med. 2011;2011:891342.

6. Ferrandon D, Imler JL, Hetru C, Hoffmann JA. The Drosophila systemic immune response: sensing and signaling during bacterial and fungal infections. Nat Rev Immunol. 2007;7(11):862-74.

7. Kim T, Kim YJ. Overview of innate immunity in Drosophila. J Biochem Mol Biol. 2005;38(2):121-7.

8. Meister S, Kanzok SM, Zheng XL, Luna C, Li TR, Hoa NT, et al. Immune signaling pathways regulating bacterial and malaria parasite infection of the mosquito Anopheles gambiae. Proc Natl Acad Sci U S A. 2005:102(32):11420-5.

9. Rutschmann S, Jung AC, Hetru C, Reichhart JM, Hoffmann JA, Ferrandon D The Rel protein DIF mediates the antifungal but not the antibacterial host defense in Drosophila. Immunity. 2000;12(5):569-80.

10. Mitri C, Jacques JC, Thiery I, Riehle MM, Xu J, Bischoff E, et al. Fine pathogen discrimination within the APL1 gene family protects Anopheles gambiae against human and rodent malaria species. PLoS Pathog. 2009;5(9): e1000576.

11. Dong Y, Dimopoulos G. Anopheles fibrinogen-related proteins provide expanded pattern recognition capacity against bacteria and malaria parasites. J Biol Chem. 2009;284(15):9835-44.

12. Riehle MM, Xu J, Lazzaro BP, Rottschaefer SM, Coulibaly B, Sacko M, et al. Anopheles gambiae APL1 is a family of variable LRR proteins required for Rel1-mediated protection from the malaria parasite. Plasmodium berghei. PLoS One. 2008;3(11):e3672.

13. Blandin S, Shiao SH, Moita LF, Janse CJ, Waters AP, Kafatos FC, Levashina EA Complement-like protein TEP1 is a determinant of vectorial capacity in the malaria vector Anopheles gambiae. Cell. 2004;116(5):661-70

14. Charroux B, Rival T, Narbonne-Reveau K, Royet J. Bacterial detection by Drosophila peptidoglycan recognition proteins. Microbes Infect. 2009;11(6-7):631-6.

15. Antonova Y, Alvarez KS, Kim YJ, Kokoza V, Raikhel AS. The role of NF-kappaB factor REL2 in the Aedes aegypti immune response. Insect Biochem Mol Biol. 2009:39(4):303-14.

16. Meister S, Agianian B, Turlure F, Relogio A, Morlais I, Kafatos FC, et al. Anopheles gambiae PGRPLC-mediated defense against bacteria modulates infections with malaria parasites. PLoS Pathog. 2009;5(8):e1000542.

17. Fivelman QL, McRobert L, Sharp S, Taylor CJ, Saeed M, Swales CA, et al. Improved synchronous production of Plasmodium falciparum gametocytes in vitro. Mol Biochem Parasitol. 2007;154(1):119-23.

18. Hoa NT, Keene KM, Olson KE, Zheng L. Characterization of RNA interference in an Anopheles gambiae cell line. Insect Biochem Mol Biol. 2003;33(9):949-57.

19. Sinden RE, Alavi $Y$, Raine JD. Mosquito-malaria interactions: a reappraisal of the concepts of susceptibility and refractoriness. Insect Biochem Mol Biol. 2004;34(7):625-9.

20. Dong Y, Das S, Cirimotich C, Souza-Neto JA, McLean KJ, Dimopoulos G. Engineered Anopheles immunity to Plasmodium infection. PLoS Pathog. 2011;7(12):e1002458

21. Warr E, Das S, Dong Y, Dimopoulos G. The Gram-negative bacteria-binding protein gene family: its role in the innate immune system of Anopheles gambiae and in anti-Plasmodium defence. Insect Mol Biol. 2008;17(1):39-51.

22. Silverman N, Zhou R, Stoven S, Pandey N, Hultmark D, Maniatis T. A Drosophila IkappaB kinase complex required for Relish cleavage and antibacterial immunity. Genes Dev. 2000;14(19):2461-71.

23. Shin SW, Kokoza V, Ahmed A, Raikhel AS. Characterization of three alternatively spliced isoforms of the Rel/NF-kappa B transcription factor Relish from the mosquito Aedes aegypti. Proc Natl Acad Sci U S A. 2002;99(15):9978-83.
24. Simoes ML, Goncalves L, Silveira H. Hemozoin activates the innate immune system and reduces Plasmodium berghei infection in Anopheles gambiae. Parasit Vectors. 2015:8:12.

25. Garver LS, Bahia AC, Das S, Souza-Neto JA, Shiao J, Dong Y, Dimopoulos G. Anopheles Imd pathway factors and effectors in infection intensitydependent anti-Plasmodium action. PLoS Pathog. 2012;8(6):e1002737.

26. Frolet C, Thoma M, Blandin S, Hoffmann JA, Levashina EA. Boosting NFkappaB-dependent basal immunity of Anopheles gambiae aborts development of Plasmodium berghei. Immunity. 2006;25(4):677-85.

27. Garver LS, Dong Y, Dimopoulos G. Caspar controls resistance to Plasmodium falciparum in diverse anopheline species. PLoS Pathog. 2009;5(3):e1000335.

28. Gupta L, Molina-Cruz A, Kumar S, Rodrigues J, Dixit R, Zamora RE, BarillasMury $\mathrm{C}$. The STAT pathway mediates late-phase immunity against Plasmodium in the mosquito Anopheles gambiae. Cell Host Microbe. 2009; 5(5):498-507.

29. Bahia AC, Kubota MS, Tempone AJ, Araujo HR, Guedes BA, Orfano AS, et al. The JAK-STAT pathway controls Plasmodium vivax load in early stages of Anopheles aquasalis infection. PLoS Negl Trop Dis. 2011;5(11):e1317.

30. Mead EA, Li M, Tu Z, Zhu J. Translational regulation of Anopheles gambiae mRNAs in the midgut during Plasmodium falciparum infection. BMC Genomics. 2012;13:366.

31. Paul RE, Bonnet S, Boudin C, Tchuinkam T, Robert V. Aggregation in malaria parasites places limits on mosquito infection rates. Infect Genet Evol. 2007; 7(5):577-86

32. Rutledge LC, Gould DJ, Tantichareon B. Factors affecting the infection of anophelines with human malaria in Thailand. Trans R Soc Trop Med Hyg. 1969;63(5):613-9.

33. Chen G, Kronenberger P, Teugels E, De Greve J. Influence of RT-qPCR primer position on EGFR interference efficacy in lung cancer cells. Biol Proced Online. 2011:13:1.

34. Shepard AR, Jacobson N, Clark AF. Importance of quantitative PCR primer location for short interfering RNA efficacy determination. Anal Biochem. 2005;344(2):287-8.

35. Holmes K, Williams CM, Chapman EA, Cross MJ. Detection of siRNA induced mRNA silencing by RT-qPCR: considerations for experimental design. BMC Res Notes. 2010;3:53.

36. Luna $\mathrm{C}$, Hoa NT, Lin H, Zhang L, Nguyen HL, Kanzok SM, Zheng L. Expression of immune responsive genes in cell lines from two different Anopheline species. Insect Mol Biol. 2006;15(6):721-9.

37. Carrara VI, Lwin KM, Phyo AP, Ashley E, Wiladphaingern J, Sriprawat K, et al. Malaria burden and artemisinin resistance in the mobile and migrant population on the Thai-Myanmar border, 1999-2011: An observational study. Plos Med. 2013;10:3.

38. Dondorp AM, Nosten F, Yi P, Das D, Phyo AP, Tarning J, et al. Artemisinin resistance in Plasmodium falciparum malaria. N Engl J Med. 2009;361(18):1808.

39. Pike A, Vadlamani A, Sandiford SL, Gacita A, Dimopoulos G. Characterization of the Rel2-regulated transcriptome and proteome of Anopheles stephensi identifies new anti-Plasmodium factors. Insect Biochem Mol Biol. 2014:52:82-93.

\section{Submit your next manuscript to BioMed Central and we will help you at every step:}

- We accept pre-submission inquiries

- Our selector tool helps you to find the most relevant journal

- We provide round the clock customer support

- Convenient online submission

- Thorough peer review

- Inclusion in PubMed and all major indexing services

- Maximum visibility for your research

Submit your manuscript at www.biomedcentral.com/submit 